\title{
Nordiques
}

36 | 2018

Réformer l'éducation en Europe du Nord

\section{Entre débats nationaux et guerre froide : réformer l'école primaire égalitaire en Finlande dans les années 1960 et 1970}

\section{Ville Okkonen}

\author{
(2) OpenEdition \\ Journals \\ Édition électronique \\ URL : http://journals.openedition.org/nordiques/504 \\ DOI : 10.4000/nordiques.504 \\ ISSN : 2777-8479 \\ Éditeur : \\ Association Norden, Bibliothèque de Caen la mer \\ Édition imprimée \\ Date de publication : 1 novembre 2018 \\ Pagination : 45-58 \\ ISBN : 9791095914020 \\ ISSN : $1761-7677$ \\ Référence électronique \\ Ville Okkonen, «Entre débats nationaux et guerre froide : réformer l'école primaire égalitaire en \\ Finlande dans les années 1960 et 1970 », Nordiques [En ligne], 36 | 2018, mis en ligne le 31 octobre \\ 2020, consulté le 15 mars 2021. URL : http://journals.openedition.org/nordiques/504 ; DOI : https:// \\ doi.org/10.4000/nordiques.504
}




\section{Entre débats nationaux et guerre froide : réformer l'école primaire égalitaire en Finlande dans les années 1960 et 1970}

Ville Okkonen*

\section{RÉSUMÉ}

1968 vit la mise en place par le Parlement finlandais d'un nouveau système d'école publique. Ce nouveau système remplaça une scolarité traditionnelle où le classement des élèves tendait à reproduire les divisions sociales. Dans cet article, nous analysons dans une perspective historique la résistance à cette réforme, en replaçant celle-ci dans le cadre de la guerre froide et de l'histoire finlandaise. Elle est analysée comme une expression de la droite conservatrice finlandaise, que nous trouvons ici motivée essentiellement par la peur de la gauche et du socialisme. Le nouveau système scolaire était vu comme une tentative de la gauche d'acquérir plus de pouvoir et de détruire la société traditionnelle. Les conséquences de la guerre civile de 1918 avaient tenu la gauche éloignée de certaines parties de la société finlandaise, en particulier la haute administration, les écoles et les universités. Le débat sur l'éducation des années 1950-1960 montre un affaiblissement de la domination de la droite dans ces secteurs, alors que la gauche renforçait sa position au Parlement, au gouvernement, dans les cercles culturels et dans ceux de l'expertise légale et sociale. La droite considéra ce retour de la gauche, et en particulier la lutte pour le nouveau système d'école publique et le nouveau statut des écoles privées, comme une attaque contre l'ordre social traditionnel.

\section{ABSTRACT}

The parliament of Finland decided on the comprehensive school in 1968. It meant a big breakage from the traditionalistic, school system that reproduced class cleavages. In this article, the resistance to comprehensive school reform is analyzed from a historical perspective. How national history and of the Cold War appeared in the political conflicts of the school reform. The rightist and conservative resistance to the comprehensive school is explained with the historical analysis of the reform process and surrounding debates. According to the research results, the resistance to the comprehensive school was before anything motivated by the fear of the left and socialism. The comprehensive school was seen as an insidious mechanism for the left to gradually take power and unsettle the traditional social order. The aftermath of the Finnish Civil War (1918) had sealed the left's expulsion from upper administration, schools and universities. This marginalization began to stagger in the 1960s when the left strengthened its position in parliament, government and in those cultural institutions in which it had been weak or without representation. The right considered that carrying out the municipal comprehensive school reform and winding up the private schools signified the growth of the influence of the left. The concept of socialism was understood broadly as a gradual erosion of the traditional social order.

\footnotetext{
* Université de Turku, Finlande.
} 


\section{INTRODUCTION}

Lécole primaire finlandaise, caractérisée par un système d'enseignement primaire polyvalent et obligatoire d'une durée de neuf ans, a suscité beaucoup d'intérêt journalistique, scientifique et politique ${ }^{1}$. Les études PISA, menées par l'OCDE depuis une quinzaine d'années et mesurant les connaissances pluridisciplinaires, ont attiré l'attention sur l'école primaire finlandaise et fait l'éloge de son éducation égalitaire. Les études historiques récentes ont pourtant démontré que ce modèle a, lors de sa création, provoqué des controverses furieuses durant lesquelles ses détracteurs envisageaient l'avenir de ce système de manière très pessimiste $^{2}$. Cet article examine d'une façon synthétique les débats politiques autour de ce projet d'école primaire en Finlande, en s'appuyant sur des recherches déjà existantes. Il s'interroge sur la manière dont cette controverse s'est inscrite dans les débats de la période de guerre froide, poursuivant les discussions de l'entredeux-guerres sur le sujet ${ }^{3}$.

En Finlande, l'élaboration d'un système d'école primaire unifié commença à la fin des années 1940. Jusque-là, le système scolaire finlandais suivait le modèle de la pohjakoulu, fixé à la fin du XIX siècle ${ }^{4}$. Dès la période de l'entre-deux-guerres, les défenseurs de l'école primaire dénonçaient la sélection précoce et la répartition des élèves en bonnes et moins bonnes écoles du système de la pohjakoulu. Ils y voyaient la marque d'une société de classes que la démocratisation du reste de la société n'avait pas encore affectée. Les défenseurs de l'école primaire égalitaire considéraient donc que les possibilités d'éducation restaient trop dépendantes de la classe sociale, de la position géographique et du patrimoine culturel jouant sur le choix de l'école . Mais ce n'est qu'en 1968 que la nouvelle loi sur l'école primaire remplaça l'ancien système. Le nouveau système (peruskoulu) n'opérait pas de sélection des élèves avant l'âge de 11 ans et faisait entrer dans leur éducation des matières plus académiques ${ }^{6}$. Politiquement, ce système de peruskoulu allait

1 Pasi Sahlberg, Suomalaisen koulun menestystarina ja mitä muut voivat siitä oppia, Helsinki, Into Kustannus Oy, 2015.

2 Ville Okkonen, Peruskoulua vastaan - yksityisoppikoulut ja yhteiskuntajärjestys 1966-1975, Turun yliopiston julkaisuja, Turun yliopisto, 2017.

3 Sur la dimension internationale de ces débats sur la mémoire de la guerre froide : Allen Hunter, Rethinking the Cold War, Philadelphie, Temple University Press, 1998, p. 2-5, p. 187-188.

4 Le système de pohjakoulu était une tentative de réformer le système scolaire créé au mitan des années 1800, en allongeant la période de scolarité commune des différentes classes de la société finlandaise. Saara Tuomaala, "Kamppailu yhteisestä koulusta ja oppivelvollisuudesta ", in Valistus ja koulunpenkki. Kasvatus ja koulutus Suomessa 1860-luvulta 1960-luvulle, A. Heikkinen et P. Leino-Kaukinen (éd.), Helsinki, Suomalaisen Kirjallisuuden Seura, 2011, p. 109.

5 Ville Okkonen, Peruskoulua vastaan, op. cit., p. 16-17.

6 Rosemary C. Salomone, "Charting a Constitutional Course between Private Values and Public Commitments : The Case of School Vouchers ", in School Choice. The Moral Debate, A. Wolfe (éd.), Princeton, Princeton University Press, 2003, p. 244-270. 
surtout à l'encontre de l'idée, partagée par les partis de droite, que les parents devaient garder le droit de choisir une école " convenable " pour leurs enfants.

Au début du $\mathrm{XX}^{\mathrm{e}}$ siècle, le projet d'école primaire égalitaire resta longtemps confiné aux marges politiques du fait de son association avec les milieux libéraux et de gauche ${ }^{7}$. Depuis la guerre civile de 1918, les intellectuels, l'administration, les experts pédagogues et l'institution scolaire cultivaient en effet une opinion négative de la gauche ; après la Seconde Guerre mondiale, la guerre froide réveilla dans un premier temps ce fantasme des "dangers de la gauche " et d'une dislocation d'un ordre sociétal porté par la tradition. Du point de vue de la droite traditionnelle, la politique scolaire de gauche était un affront aux valeurs culturelles et nationales et à l'état naturel de la société. L'école était même vue dans ces milieux comme secondaire par rapport à d'autres institutions (armée, église, associations de défense volontaires, etc.) considérées comme plus importantes pour maintenir l'ordre social. L'école municipale publique semblait donc menacer autant la propriété privée que les opinions établies aux yeux de cette droite conservatrice ${ }^{8}$. On peut noter qu'aux États-Unis, la droite partageait des opinions semblables sur l'institution scolaire?.

Il convient ici de s'arrêter quelques instants sur les définitions propres au contexte finlandais des termes " droite ", " gauche » et " centre ». Ils sont en effet largement dépendants de leurs contextes chronologique et géographique. Dans cet article, droite et gauche désignent des groupements d'individus réunis par une culture politique plus large que le cercle des adhérents à certains partis politiques : on y trouve aussi bien des hommes et femmes politiques que des acteurs culturels, des intellectuels et des fonctionnaires. Pour la gauche, elle se définit à partir de 1918 autour du parti social-démocrate, du parti communiste et d'un groupe socialiste de gauche situé entre les deux. Dans le cadre de la politique scolaire, ces groupes sont unanimes dans leur soutien à une égalité accrue dans le système scolaire et à la fin de la société de classes. Après la Seconde Guerre mondiale, la gauche communiste aussi défendit de façon très affirmée ces idées. Le terme de "droite " recouvre les partis Kokoomus (droite conservatrice), RKP (Parti du peuple suédois) et la frange droite du parti du centre (Ligue agraire), de même

7 Lars Björne, "....syihin ja lakiin eikä mielivaltaan... " Tutkimus Turun hovioikeuden poliittisista oikeudenkäynneistä vuosina 1918-1939, Helsinki, Suomalainen lakimiesyhdistys, 1977, p. 24, p. 99 ; Jukka Kekkonen, Suomen oikeuden historiallisia kehityslinjoja, Helsinki, Helsingin yliopisto, 1999, p. 82.

8 Ville Okkonen, Peruskoulua vastaan, op. cit., p. 13, p. 63, p. 311.

9 Adam Laats, The Other School Reformers. Conservative Activism in American Education, Cambridge, Harvard University Press, 2015, p. 3 ; Åke Islingin, Kampen for och mot en demokratisk skola. Samhällsstruktur och skolorganisation, Stockholm, Sober, 1980 ; Andrew Hartman, Education and the Cold War. The Battle for the American School, New York, Palgrave Macmillan, 2008 ; Oona Ilmolahti, Eheys ja ennakkoluulo. Työväenyhteisön ja kansakoulunopettajiston jännitteinen subde Helsingissä sisällissodasta 1930-luvulle, Helsinki, Helsingin yliopisto, 2017. 
que dans les années 1960-1970 le Parti rural finlandais (SMP). Ce groupe est unifié par son conservatisme, qui se traduit par une volonté de préserver une société " naturelle » « héritée des ancêtres » (l'expression « isiltä peritty», héritée des pères, devenant un slogan souvent répété dans les débats). Il souhaite préserver les résultats de la victoire des Blancs en $1918^{10}$ et faire front au "marxisme ». Ce groupe est toutefois divisé, puisque sa frange la plus libérale se distingue par son analyse de la société et sa proximité dans les questions culturelles et d'éducation avec les sociaux-démocrates ou même les communistes.

\section{UNE CONCEPTION RÉACTIONNAIRE DES INSTITUTIONS CULTURELLES}

Dans cette lutte à propos de l'école primaire égalitaire, la radicalité des positions se nourrissait d'une longue histoire. La guerre civile de 1918 et ses répercussions avaient fait de l'administration de l'État et des institutions intellectuelles des organismes très conservateurs. La révolution bolchévique en Russie avait décomposé le mouvement ouvrier et poussé le corps électoral finlandais vers la droite. Or celle-ci se voyait comme l'héritière de la victoire des Blancs lors de la "guerre de libération " de 1918, qui inspirait chez elle un rejet instinctif de tout ce qui ressemblait au socialisme. Dans les années 1920, plusieurs lois proposées par le centre et la gauche, reconnaissant les divisions sociales de la société et cherchant à les résoudre (notamment la loi sur la scolarité obligatoire en 1921), apparaissaient à cette droite conservatrice comme une dépense irresponsable de fonds publics et un paternalisme malsain. Pour la gauche, l'expansion de l'institution scolaire et la scolarité obligatoire étaient autant de moyens d'abolir la société de classes. Pour les libéraux, il s'agissait de renforcer la cohésion sociale et le système constitutionnel républicain. Malgré plusieurs propositions de modèle scolaire libertaire et socialiste, le système scolaire de la fin du XIX ${ }^{e}$ siècle resta toutefois en vigueur ${ }^{11}$.

Il est important de noter ici la façon dont l'indépendance finlandaise a pu être divisée en deux séquences, qu'on a appelées "première république " et " deuxième république ». Marquée par la mise en place d'une constitution républicaine et les épreuves de la guerre, la première république s'arrête en 1945, laissant la place à un contexte de construction de l'État providence, de solidification de l'État de droit malgré la guerre froide et d'une société transformée en profondeur par l'éducation de masse. Cette idée de deux "républiques " ${ }^{12}$ et la façon de les

10 Entre janvier et mai 1918, la Finlande se déchire au cours d'une guerre civile suivant le modèle de la guerre civile russe : les Blancs conservateurs se voient opposés aux Rouges représentants d'une frange radicalisée du mouvement ouvrier.

11 Ville Okkonen, Peruskoulua vastaan, op. cit., p. 39-43.

12 Martti Ahti, "Tanners minoritetsregering, skyddskårerna och "fascismens spöke" ", Historisk Tidskrift för Finland, 4, 1984 ; Etelä-Suomen Sanomat, 13 novembre 1974 ; Maaseudun Tulevaisuus, 31 mai 1969. 
définir ont été mises en avant par l'essayiste Matti Kurjensaari, lorsqu'il écrit que, si la nation s'imposa à la société en 1918, la société s'imposa à la nation après 1944. La première république était ainsi dominée par une vision extrêmement stricte et exclusive de la nation propagée par des intellectuels conservateurs et nationalistes, fondée sur l'héritage de la victoire blanche de 1918. Cette narration nationale dominante s'appuyant sur le souvenir de la guerre civile, la gauche politique se trouvait privée d'une grande partie de sa marge de manœuvre symbolique et politique ${ }^{13}$.

Matti Kurjensaari, toutefois, prend en compte un certain délai dans le changement politique en Finlande, soulignant que le tournant de 1944-1945 ne se manifesta véritablement que dans les années 1960 et 1970, alors que la génération de l'entre-deux-guerres se retirait des postes importants et laissait la place à une génération désireuse de réformer en profondeur les institutions scolaires et culturelles ${ }^{14}$. La recherche historique a maintes fois montré que les dirigeants finlandais de l'immédiat après-guerre trouvaient encore leurs inspirations dans le contexte intellectuel de l'entre-deux-guerres ${ }^{15}$. En outre, ils avaient tendance à considérer comme "marxiste " toute pensée sociale, et comme antinationale toute tentative de réforme ${ }^{16}$.

Mais le naufrage de la Seconde Guerre mondiale, qui se termina par une défaite finlandaise, l'armistice de Moscou en 1944, et des élections législatives en 1945 qui renforcèrent la gauche, aboutit à une vie parlementaire dominée momentanément par la gauche et ouvrit le champ à une possible réforme du système scolaire. Le communisme et le socialisme de gauche furent légalisés, et une nouvelle direction politique se trouva représentée au Parlement. On engagea ainsi une réforme totale de l'institution scolaire, dirigée par le professeur antimilitariste Yrjö Ruutu. Ce dernier avait en tête un ensemble de réformes auparavant impossibles du fait des rapports de force politiques, mais aussi de l'opposition d'une fraction importante des élites finlandaises, en particulier les cercles cléricaux et les pédagogues les plus conservateurs.

Les débuts de la « deuxième république » virent donc un changement radical dans la politique intérieure et extérieure finlandaise. De nouveau, Kurjensaari donne la meilleure expression de ce nouvel esprit, demandant que la Finlande devienne nationalement et socialement plus dynamique, plus prospère et qu'elle se reconstruise sur des fondements intellectuels différents de ceux d'avant-guerre.

13 Antero Jyränki, Kansa kahtia, henki halpaa. Oikeus sisällissodan Suomessa?, Helsinki, Art House, 2014, p. 218-219, p. 224.

14 Matti Kurjensaari, Syntynyt sivulliseksi. Näkyjä ja näkemyksiä 1960-1973, Helsinki, WSOY, 1973, p. 217.

15 Matti Klinge, Vihan veljet ja kansallinen identiteetti, Helsinki, Siltala, 2012, p. 170.

16 Martti Ahti, «Frontmän på högerkanten », Historisk Tidskrift för Finland, 2, 1989, p. 353. 
Il considérait comme la gauche que cette reconstruction demandait une introspection nationale, une réflexion sur l'identité du pays et donc la réforme du système scolaire et culturel ${ }^{17}$. De la sorte, cette réforme du système scolaire était bien plus qu'une question technique : une question d'identité nationale influençant les structures de la société, la politique étrangère, et la relation des Finlandais à leur histoire.

Mais dans cet immédiat après-guerre, malgré les victoires électorales de la gauche et l'influence de l'URSS, la grande majorité des intellectuels et des enseignants ne ressentait aucun besoin d'autocritique ou de réappréciation du passé national. Dans ce domaine, le professeur d'école Toivo Lähdesmäki exprime bien une certaine anxiété de la gauche dans son discours durant une conférence de la société Finlande-URSS (Suomi-Neuvostoiitto-Seura). Lähdesmäki y remercie la société de son soutien dans la lutte pour un nouvel enseignement ${ }^{18}$. L'extrême gauche voyait ce combat comme une continuation des débats de l'entre-deuxguerres, où les sociaux-démocrates et les libéraux de droite avaient défendu de nouvelles formes d'enseignement contre le conservatisme dominant. Le journal de gauche Työkansan Sanomat rappelait ainsi le 21 janvier 1948 le travail des directeurs successifs de l'administration scolaire, Mikael Soininen et Oskari Mantere, tous deux de droite, mais ayant œuvré pour la réforme scolaire ${ }^{19}$. Le débat n’avait donc pas commencé avec la guerre, pas plus qu'il n'était le résultat d'une quelconque conspiration communiste, mais trouvait ses racines dans les polémiques de l'entre-deux-guerres.

Or l'enthousiasme portant cette réforme totale perdit vite ses fondements politiques quand l'Europe de l'Est tomba dans le giron soviétique et que la gauche finlandaise se divisa, empêchant la réforme scolaire de se réaliser et poussant les communistes et les socialistes dans l'opposition. Ce n'est qu’à la fin des années 1950 que les circonstances devinrent de nouveau favorables à une collaboration entre le centre et la gauche.

À ce moment, les communistes commencèrent à mettre en cause leur ligne stalinienne rejetée par les intellectuels ${ }^{20}$. Le parti communiste étant entre 1958 et 1962 le plus grand parti finlandais, le gouvernement put - en s'appuyant sur lui réaliser plusieurs réformes sociales, dont celle de l'impôt. En 1959, la gauche dans son ensemble (communistes et sociaux-démocrates) proposa la municipalisation des écoles primaires et la réalisation d'un système d'enseignement égalitaire, un

17 Matti Kurjensaari, écrit non daté, dossier 9, Papiers Kurjensaari, Suomalaisen Kirjallisuuden Seura.

18 Työkansan Sanomat, 9 janvier 1948.

19 Työkansan Sanomat, 21 janvier 1948.

20 Ville Okkonen, Peruskoulua vastaan, op. cit., p. 43-47. 
projet que la droite critiqua comme contraire à l'ordre naturel des sociétés qu'elle se proposait de défendre. Cette résistance trouva surtout son expression dans les cercles les plus conservateurs de spécialistes de la question scolaire, mais ces derniers ne pouvaient rien contre une évolution des rapports de force politiques qui faisait de l'idée d'école primaire égalitaire un compromis acceptable par la gauche et le centre, susceptible de remettre en question l'esprit conservateur de l'institution scolaire ${ }^{21}$.

L'évolution de l'école primaire en Finlande se trouvait au demeurant justifiée par les réformes scolaires mises en place dans le reste de l'Europe, motivées par la mobilité sociale et l'égalité éducative ${ }^{22}$. En effet, les années 1960-1970 en Occident sont fréquemment considérées comme une période de crise du modèle familial traditionnel, car l'expansion de l'État providence affaiblissait les mécanismes de reproduction sociale, remettait en cause l'institution familiale et les rôles genrés, tout en affectant la place des parents dans les décisions pédagogiques ${ }^{23}$. En Finlande, les défenseurs de l'école primaire s'inspiraient surtout du modèle de la Suède, où le développement de l'État providence et de l'école primaire était bien plus avancé. Dans les années 1960, certains d'entre eux adoptèrent aussi un mode de pensée plus individualiste qui mettait en question les rôles au sein de la famille et le pouvoir des parents sur le choix de l'école ${ }^{24}$.

Les générations du baby-boom nées entre les années 1950 et 1960 augmentaient aussi le besoin d'éducation. Celle-ci se généralisait et l'institution scolaire perdait son caractère élitiste. En 1960, il était clair que l'ancien système scolaire touchait à sa fin, et les partis de gauche et du centre apportèrent leur soutien à la réforme scolaire. En 1963, avec l'appui de la gauche et du centre, le Parlement réagit aux atermoiements du gouvernement en pressant celui-ci de commencer à préparer une loi sur l'école primaire. Le rapport de force politique était favorable : les élections législatives de 1966 venaient de donner une majorité aux partis de gauche qui avaient formé un gouvernement de front populaire (kansanrintamahallitus) avec le centre. Les démocrates populaires se retrouvèrent au gouvernement après dix-huit ans d'absence. Ces changements de politique intérieure mettaient au jour un corps électoral s'orientant vers la gauche ${ }^{25}$.

21 Ibid., p. 50 .

22 Geoffrey Waldorf, Diversity an choice in school education. An alternative view. Markets and Equity in Education, New York, Continuum, 2006, p. 82-83.

23 Eric Hobsbawm, Äärimmäisyyksien aika. Lyhyt 1900-luku (1914-1991), Tampere, Vastapaino, 2014, 407, 409 .

24 Gøsta Esping-Andersen, "Towards the Good Society, Once Again ?", in Why we need a New Welfare State, G. Espin-Andersen, D. Gallic, A. Hemerijck et J. Myles (éd.), Oxford, Oxford University Press, 2002, p. 13.

25 Ville Okkonen, Peruskoulua vastaan, op. cit., p. 76-81. 
Ce tournant dans la politique intérieure fut vécu comme un cauchemar par la droite qui eut l'impression d'une victoire du socialisme et d'une défaite des valeurs traditionnelles (patriotisme, institution scolaire, histoire nationale...). Dans le contexte de la guerre froide, un gouvernement comprenant l'extrême gauche était à leurs yeux un sujet de honte : le communisme représentait pour le bloc de l'Ouest la conspiration absolue, le contraire du pluralisme et un pouvoir antidémocratique. La droite interprétait aussi la perte de sa position dominante comme une " politisation » des débats de société. Dans les cercles qu'elle influençait, la gauche était perçue comme un mouvement strictement partisan, hostile à l'état " naturel » de la société et bouleversant à la fois le rôle et la composition de ses élites ${ }^{26}$. Malgré l'importance en toile de fond du contexte de guerre froide, cette défense des privilèges de l'élite et d'une société " d'ordre " pesa plus lourd dans les réactions de la droite que la peur géopolitique d'une extension du communisme.

Pour les conservateurs, la gauche s'attachait en particulier à " politiser » les institutions et les valeurs culturelles traditionnelles ${ }^{27}$. Cela intensifiait la méfiance que la droite avait déjà envers la réforme scolaire. Les institutions culturelles représentant les idéaux conservateurs concernant la nation, auparavant monopolisés par la droite, semblaient menacées. Pour les plus conservateurs, un communiste n'était ni un Finlandais ni un patriote. Mais une certaine bourgeoisie, culturelle et libérale, avait révisé son patriotisme durant les années 1950, se démarquant de la droite dure, ce qui accéléra probablement la formation du front populaire en 1966. Les tournants de la politique intérieure, l'arrivée de la gauche au gouvernement et à la tête des institutions culturelles les plus traditionalistes (par exemple l'université d'Helsinki, les principales écoles, les prix littéraires et autres) finirent par briser l'ancien ordre social, pour qui 1918 avait été une révolte illégitime et la droite une force apolitique représentant la nation dans son ensemble. Les effets de la croissance de l'institution scolaire et de la généralisation de l'éducation devinrent évidents dans les années 1960 et 1970 lorsque l'intelligentsia de gauche commença à peupler les principales institutions culturelles. Écoliers et étudiants se montraient alors très intéressés par les idées de gauche et la jeunesse se révoltait, comme pendant l'occupation de la maison des étudiants d'Helsinki au printemps 1968, contre une droite conservatrice qui, de son côté, voyait l'opposition à la guerre du Vietnam, les mœurs libérales et le pacifisme comme autant de manifestations du communisme. À la fin des années 1950 et tout au long des

26 Juhani Suomi, Kriisien aika. Urho Kekkonen 1956-1962, Helsinki, Otava, 1992, p. 185 ; Juhani Suomi, Presidentti. Urho Kekkonen 1962-1968, Helsinki, Otava, 1994, p. 408.

27 Ville Okkonen, Peruskoulua vastaan, op. cit., p. 96-99. 
années 1960, la littérature populaire se mit aussi à défaire les mythes officiels de l'histoire nationale ${ }^{28}$.

Ces bouleversements provoquèrent une riposte de la part des cercles d'intellectuels conservateurs, qui organisèrent ainsi des séances d'éducation patriotique destinées aux professeurs et aux directeurs d'écoles. Nombre de chercheurs et professeurs influents prirent néanmoins leurs distances avec cette campagne alarmiste pour restaurer « l'ordre social » légitime. Il est possible, et probable même, que l'activisme et les opinions tranchées des conservateurs aient facilité l'évolution d'intellectuels, jeunes et plus âgés, vers la gauche, et justifié aussi un nouveau mouvement progressiste au sein des écoles et des universités.

\section{AVEC QUELS ARGUMENTS S'OPPOSER À LA RÉFORME DE L'ÉCOLE ?}

Dans ces débats, la droite modelait son argumentaire en particulier autour du droit des parents à décider de la qualité et du contenu de l'éducation de leurs enfants. Cela signifiait la sélection précoce des élèves et l'existence d'écoles primaires garanties par le droit de la propriété privée. Mais bien que ces écoles fussent aussi dépendantes financièrement des fonds publics, le projet d'école primaire municipale représentait pour la droite traditionnelle une pression socialiste partisane sur la liberté des familles : les parents devaient continuer à être les meilleurs connaisseurs des capacités de leurs enfants et les écoles privées devaient rester à l'écart de la démocratie municipale et de la sphère publique. Pour la droite traditionnelle, les écoles privées représentaient une culture et des traditions facilitant les demandes des parents et la reconnaissance des capacités des enfants ${ }^{29}$.

Pour combattre l'école primaire égalitaire, les activistes défendaient les écoles privées en Finlande et partout dans les pays nordiques en invoquant la Déclaration universelle des droits de l'homme des Nations unies (1948), le Pacte international relatif aux droits économiques, sociaux et culturels (1966), les pactes du Conseil de l'Europe, la Convention européenne des droits de l'homme et la Déclaration sur l'éducation de l'UNESCO en 1960. Ces documents, largement déclaratifs, pouvaient être interprétés de différentes manières comme argumentaire en faveur de l'ancien système scolaire. Ils étaient utilisés comme sources

28 Ibid., p. 152-153.

29 Amy Gutmann, "Assessing Arguments for School Choice : Pluralism, Parental Rights, or Educational Results ?", in School Choice. The Moral Debate, A. Wolfe (éd.), Princeton, Princeton University Press, 2003, p. 126-148 ; Stephen Provasnik, "Judicial Activism and the Origins of Parental Choice : The Court's Role in the Institutionalization of Compulsory Education in the United States 1891-1925 ", History of Education Quarterly, 46, 2006, History of Education Society, p. 311-347 ; Geoffrey Waldorf, Diversity an choice in school education, op. cit., p. 11, p. 13 ; Rosemary C. Salomone, "Charting a Constitutional Course between Private Values and Public Commitments ", op. cit., p. 244-270. 
justifiant le droit des parents à choisir leur école, droit menacé par ce nouveau modèle d'école publique lié à la démocratie municipale ${ }^{30}$.

Mais alors que la question de la gestion des aides publiques par les écoles privées était discutée, et que les dispositions législatives étaient extrêmement floues, les politiciens conservateurs présentaient leurs positions au Parlement et dans la presse selon une ligne purement juridique visant à sauvegarder « l'ordre social ». L'affaiblissement du monopole de la droite dans les domaines de l'expertise constitutionnelle et pédagogique affectait les discussions, surtout dans les années 1968-1974 lorsqu'une bonne partie des professeurs de droit constitutionnel $^{31}$ se trouva composée d'hommes de gauche. Des deux côtés, les objectifs politiques inspiraient les positions ${ }^{32}$. Dans la lutte pour l'école primaire, la gauche suivait sa ligne traditionnelle, protégeait la majorité gouvernementale et avançait un programme égalitariste en proportion des besoins sociaux et culturels. Elle critiquait le système constitutionnel, qui établissait la propriété privée comme droit fondamental, en limitant le pouvoir de la majorité parlementaire : les principales réformes constitutionnelles finlandaises (1906, 1919 et 1928) avaient vu le jour à des périodes où le rejet du socialisme était prévalent, et la défense de la propriété privée y était clairement inscrite ${ }^{33}$. Dans la lutte pour l'école primaire, la droite protégeait ses idéaux politiques en s'appuyant sur une argumentation majoritairement juridique. Mais cette argumentation se retrouva dans une impasse quand des professionnels du droit issus de la gauche furent en position d'interpréter la Constitution d'une autre manière ${ }^{34}$.

30 Ville Okkonen, Peruskoulua vastaan, op. cit., p. 181-186.

31 L'expertise constitutionnelle et l'interprétation de la Constitution en Finlande relèvent du domaine du Parlement, et particulièrement du comité parlementaire chargé des questions constitutionnelles, qui les étudie en consultant des experts. Il n'existe pas en Finlande l'équivalent de la Cour constitutionnelle et du Conseil d'État francais.

32 Ville Okkonen, Peruskoulua vastaan, op. cit., p. 203-204.

33 Ibid., p. 100-107.

$34 \mathrm{La}$ protection de la propriété privée étant inscrite dans la Constitution et dans les règles décisionnelles du Parlement finlandais, la réforme du système scolaire est aussi discutée en relation avec le système constitutionnel, par des experts qui, dans les années 1960, sont encore principalement des conservateurs. Cette hégémonie de la droite conservatrice en matière d'expertise constitutionnelle est toutefois remise en question dans les années 1970. Malgré cela, les opposants à l'école primaire exploitent l'argumentation constitutionnelle pour défendre le statut des écoles privées, en arguant que les écoles municipales publiques remettent en cause la position des écoles privées. Suite à la décision de 1968, l'opposition de droite au Parlement insiste donc sur le rôle du Parlement comme garantie de la protection de la propriété. Lorsque la mise en place de l'école unitaire municipale devint plus difficile, le gouvernement décida de clarifier la section relative à l'institutionnalisation de l'enseignement privé, approuvée par le Parlement à l'été 1974. En réaction, les partis de droite dénoncèrent une "politisation " de l'expertise constitutionnelle tirant le pays vers le socialisme, alors que selon eux leur position de défense de la propriété privée était liée à la défense de valeurs et de principes juridiques immanents. 


\section{L'INFLUENCE DE LA GUERRE FROIDE SUR LES DÉBATS}

En Finlande, la fin de l'URSS provoqua une réévaluation de la politique extérieure et de l'influence de ce pays. Un discours conservateur de guerre froide se développa avec des pamphlets et autres publications diabolisant la gauche, donnant une image héroïque de l'activisme contre l'influence russe, insistant sur la défense nationale comme source de préservation des valeurs finlandaises, etc. L'image simpliste du combat entre deux systèmes politiques avait un but précis dans le contexte de politique intérieure post-1992 : prouver l'excellence de l'économie de marché et soutenir les partis de droite. La droite conservatrice voulut redorer son blason et prendre une revanche, en dénonçant l'influence soviétique et en réinterprétant à sa manière les rapports de force politiques des années 1960-1970.

La lutte pour l'école primaire reste un exemple important de la compétition politique du temps de guerre froide et une preuve tangible de son caractère souvent manichéen. Les opposants à l'école primaire égalitaire n'étaient pas seulement motivés par leur rejet du socialisme, mais également par la lutte contre la marginalisation de leurs valeurs et le nouveau rôle institutionnel de la gauche, qui brisait le monopole social de fait de la droite. La droite finlandaise craignait l'URSS, mais aussi et surtout des dangers plus immédiats : le socialisme suédois et le pouvoir parlementaire de la gauche en Finlande. Bien que le maccarthysme américain se fût répandu à l'échelle internationale, en Finlande, l'opposition entre la gauche et la droite prenait sa source essentielle dans la mémoire des événements de 1918 telle que la droite l'avait formulée entre les deux guerres et dans la lutte menée contre elle par le mouvement ouvrier. D'ailleurs, lorsqu'on commença, au sein de la droite conservatrice, à s'apercevoir que les communistes n'étaient pas forcément antipatriotes, l'extrême droite resta presque seule à s'opposer à la progression de la gauche.

L'antagonisme de la guerre froide se manifestait donc partout, mais différemment d'un pays à l'autre. Il faut remettre en question l'interprétation selon laquelle, pendant la guerre froide, la droite aurait été martyrisée parce qu'elle voulait défendre la démocratie. En réalité, elle souhaitait surtout dans le débat qui nous intéresse ici perpétuer l'ordre social et la vie politique d'autrefois. La conservation des écoles privées et le rejet des écoles municipales faisaient partie de cette mentalité. La droite était convaincue que la gauche souhaitait avant tout subvertir l'ordre «naturel » et ses valeurs ${ }^{35}$. Les études les plus récentes ont insisté sur le rôle des agents locaux, par exemple dans l'adoption du maccarthysme ${ }^{36}$. Le combat

35 Ibid., p. 221-224.

36 Heonik Kwon, The Other Cold War, New York, Columbia University Press, 2010, p. 19-20, p. 30, p. 143 ; Mark Mazower, After the War Was Over. Reconstructing the Family, Nation, and State in Greece, 1943-1960, Princeton, Princeton University Press, 2000, p. 16. 
pour l'école primaire démontre que les lignes idéologiques, caractéristiques de la guerre froide, s'adaptaient aux circonstances locales. Dans les pays nordiques, l'État providence adoptait une combinaison d'économie de marché libre et de socialisme, ce qui résumait le mieux aux yeux de ses adversaires « les dangers de la gauche ». En Finlande, dans les années 1950 et $1960^{37}$, la droite aussi bien que la gauche faisaient un lien entre les services publics, la répartition des revenus et le socialisme. La lutte des deux systèmes était parfois discrète et il n'existait pas un seul modèle de "monde libre ». Il est impossible de présenter l'option choisie par Barry Goldwater ou Lyndon B. Johnson comme le seul modèle occidental possible, ni les positions d'Olof Palme ou Yngve Holmberg ${ }^{38}$ comme les seuls modèles de pays nordiques.

La bipolarisation de la guerre froide pouvait prendre des formes assez feutrées en fonction du contexte historique et social national. Selon Matti Klinge, il convient d'éviter les généralisations grossières de l'image de l'Occident de cette époque à partir du capitalisme américain, du gaullisme français ou de l'État providence suédois, comme à partir des mouvements d'extrême gauche en Finlande, en Allemagne et en Italie ${ }^{39}$. Pourtant, un certain type de littérature a récemment voulu faire de la politique scolaire de la droite la seule politique "occidentale », et donc démocratique ${ }^{40}$. En fait, pour les contemporains, la démocratie et la défense de l'individu étaient sujettes à débats : ainsi Olof Palme refusait-il l'identification de l'Occident avec l'économie de marché libre, car, selon lui, les racines de son socialisme démocratique se situaient aussi dans une tradition de pensée occidentale ${ }^{41}$. La volonté de libérer l'individu des contraintes de sa position sociale est au cœur de la pensée de Palme, et on en retrouve des éléments dans le débat sur l'école en Finlande, mais aussi dans le reste de l'Europe.

La peur du socialisme suédois, qui marqua ces débats dans les années 1960 et 1970, était elle aussi ancienne en Finlande. En 1938, la députée ultra-conservatrice et directrice d'école Hilja Riipinen prévenait ses compatriotes de l'influence " occidentale », dans laquelle elle voyait sous les dehors de la social-démocratie " peralbinhanssonienne "(de Per Albin Hansson, le dirigeant des sociauxdémocrates suédois et Premier ministre de l'époque) une forme du marxisme et de

37 Päivi Uljas, "Iso paha julkinen sektori », Kulttuurivihkot, 2, 2015.

38 Holmberg était un homme politique suédois, dirigeant de la droite conservatrice dans les années 1950-1960.

39 Matti Klinge, Onko omaisuus varkautta ? Päiväkirjastani 2008-2009, Helsinki, Siltala, 2009, p. 140.

40 Jouko Teperi, "Yksityisten oppikoulujen taistelu olemassaolostaan ", in Yksityisoppikoulujen historia 1872-1977, J. Salminen, J.-P. Pietiäinen et J. Teperi (dir.), Helsinki, Painatuskeskus Oy, 1995, p. 251.

41 Olof Palme, Socialdemokraterna partikongressen 1975, Olof Palme arkiv: http://www.olofpalme. org/wp-content/dokument/750928b_sap.pdf. 
l'Internationale rouge ${ }^{42}$. Après la guerre, la presse aussi bien communiste que sociale-démocrate insista en revanche sur les exemples étrangers de réforme scolaire, en particulier suédois, britannique, américain et soviétique. Dans le journal Vapaa Sana, le ministre de l'Éducation d'extrême gauche Johan Helo proposait ainsi le 4 août 1945 que la Finlande s'inspire plus clairement dans sa politique scolaire des politiques nordiques ${ }^{43}$. Il proposa de même que les ministres nordiques de l'Éducation se rencontrent régulièrement. En outre, il estimait (de façon peut-être un peu optimiste ${ }^{44}$ ) que les milieux académiques finlandais étaient en train de s'éloigner des influences allemandes et des contacts de l'époque du nazisme. Dans ces débats, l'idée d'une réforme scolaire se mêlait à ceux sur le socialisme international et apparaissait comme une tentative de mettre aussi de côté le groupe des experts pédagogiques traditionnels, liés aux forces politiques conservatrices. Cette tentative était vue à droite avec une certaine terreur, et on y répondait en rejetant les influences étrangères. L'éditorialiste anonyme Aku écrivait ainsi dans le journal Etelä-Suomen Sanomat, le 2 juin 1950, que la Finlande ne devait pas suivre les modèles étrangers : "Le système scolaire doit être conçu dans un lien organique avec les réalités et le développement de la nation. ${ }^{45}$

\section{Conclusion}

L'opposition idéologique à l'école primaire égalitaire fait partie intégrante d'une tradition conservatrice en Finlande, cherchant à défendre ce qu'elle considère comme un état « naturel » de la société et de ses hiérarchies. Les controverses sur la politique scolaire dans les années 1960 rappelaient les dissensions nées à la fin du XIX siècle à propos de l'école traditionnelle et de la scolarité obligatoire. L'immédiat après-guerre vit se démanteler la collaboration entre partis de gauche sur la question et poussa pour longtemps les communistes dans l'opposition, laissant les domaines prestigieux de l'école, de la culture et de l'université aux mains de la droite jusque dans les années 1960. Tout au long de cette décennie et à la suite d'importants tournants en politique intérieure, la réforme de l'école primaire coïncida aussi avec une phase au cours de laquelle la tradition de la Finlande «blanche » de droite fut remise en question dans les institutions culturelles et où l'intelligentsia de gauche gagna du terrain dans les universités et les organismes d'État.

42 Hilja Riipinen, "Orientoidutaan länteen », Uusi Huomen, 30 juillet 1938, p. 6.

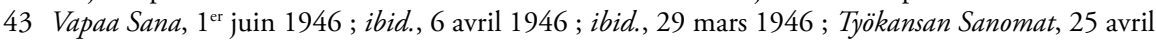
1946 ; Vapaa Sana, 4 août 1945.

44 Les élites finlandaises ont eu, depuis l'indépendance du pays en 1917, d'intenses relations culturelles avec l'Allemagne de Weimar, puis avec l'Allemagne nazie. Sur ces contacts, voir en particulier Vesa Vares, Viileää veljeyttä, Helsinki, Otava, 2018.

45 Etelä-Suomen Sanomat, 2 juin 1950. 
Le droit des parents de choisir la meilleure éducation pour leurs enfants devint alors l'objet du débat le plus ardent en Finlande. Ce combat autour du système scolaire se déroula donc à plusieurs niveaux : un niveau national où les polémiques de l'entre-deux-guerres se perpétuaient, et un niveau international marqué par la guerre froide, les deux étant bien évidemment liés. On manifesta à droite une méfiance envers la démocratie municipale, qui instituait l'école primaire égalitaire et la municipalisation des écoles privées. Le rejet de cette influence de gauche était au cour de l'opposition à l'école primaire. La droite conservatrice était ébranlée par la disparition des idéaux traditionnels concernant la famille. Les communistes obtenaient à leur tour des postes à l'université et l'opinion publique commençait de plus en plus à voir la question scolaire comme une possibilité de mettre fin au monopole culturel de la droite. Du point de vue des conservateurs, la société devenait plus imprévisible comme des personnalités de gauche pouvaient désormais devenir experts et diriger des organismes publics. Pour la gauche et pour les bourgeois libéraux, au contraire, l'école traditionnelle procédait d'une utopie individualiste dans laquelle l'individu était comme affranchi des limites de la famille, de l'ethnicité ou des opinions politiques. La droite conservatrice voulait, quant à elle, préserver les écoles privées et la sélection scolaire. Elle voyait l'école municipale comme un gâchis de ressources et une baisse de la qualité de l'éducation. Les études successives ont montré que ces peurs étaient pour le moins exagérées, voire sans fondement : au contraire, le système d'école primaire adopté a permis une amélioration des capacités cognitives de la population et facilité la mobilité sociale ${ }^{46}$.

46 Tuomas Pekkarinen, Roope Uusitalo et Sari Kerr, «School tracking and intergenerational income mobility. Evidence from the Finnish comprehensive school reform ", Journal of Public Economics, 93, 2009, p. 965-973. 